\title{
Water-compatible Cycloadditions of Oligonucleotide-conjugated Strained Allenes for DNA-encoded Library Synthesis
}

\author{
Matthias V. Westphal, ${ }^{[a, c]}$ Liam Hudson, ${ }^{[a, c]}$ Jeremy W. Mason, ${ }^{[a, c]}$ Frédéric J. Zécri, ${ }^{*}{ }^{,[c]}$ Karin Briner, ${ }^{*},[c]$ Stuart L. \\ Schreiber ${ }^{*},[a, b]$ \\ [a] Chemical Biology and Therapeutics Science Program, Broad Institute, 415 Main Street, Cambridge, MA 02142, USA \\ [b] Department of Chemistry and Chemical Biology, Harvard University, 12 Oxford Street, Cambridge, MA 02138, USA \\ [c] Novartis Institutes for BioMedical Research, 181 Massachusetts Avenue, Cambridge, MA 02139, USA
}

\begin{abstract}
DNA-encoded libraries of small molecules are being explored extensively for the identification of binders in early drug-discovery efforts. Combinatorial syntheses of such libraries require water- and DNAcompatible reactions, and the paucity of these reactions currently limit the chemical features of resulting barcoded products. The present work introduces strain-promoted cycloadditions of cyclic allenes under mild conditions to DNA-encoded library synthesis. Owing to distinct cycloaddition modes of these reactive intermediates with activated olefins, 1,3-dipoles and dienes, the process generates diverse molecular architectures from a single precursor. The resulting DNA-barcoded compounds exhibit unprecedented ring and topographic features-related to elements found to be powerful in phenotypic screening.
\end{abstract}

The identification of small molecules that bind biological macromolecules is a key step in early drug discovery. Target-directed, binding-based approaches include high-throughput, fragment, and in silico screening. While each of these techniques has had significant impact on successful drug development programs, they also have shortcomings. An additional approach uses DNA-encoded libraries (DELs), which comprise collections of compounds individually barcoded with DNA sequences that report on the synthetic reactions leading to their formation. ${ }^{[1]}$ DELs are typically prepared by split-andpool synthesis from central scaffolds and readily available building blocks as appendages. DEL screens are commonly performed using immobilized proteins, and barcode enrichment, as a surrogate for binding, is determined by next-generation sequencing of PCR-amplified DNA. DELs are a promising source of hit compounds with several examples having advanced to clinical candidates. ${ }^{[2]}$ Beyond affinitybased screens, recent work relying on spatial separation of individual library members by microfluidics hint that DELs may become amenable to activity-based screens. ${ }^{[3,4]}$ As for all hit-finding approaches, compound libraries spanning diverse chemical space with features wellsuited for binding are considered most promising, ${ }^{[5]}$ especially in the absence of known target binders.

Diversity-oriented synthesis (DOS) has been particularly successful in generating structure-diverse, stereochemistry-rich libraries that include many distinct, rigid ring skeletons that can reduce the entropic cost of binding and effectively display appendages in three-dimensional space. ${ }^{[6]}$ Often fueled by advancements in reaction methodology, the application of DOS principles has delivered numerous chemical probes and clinical candidates. ${ }^{[7]}$ Merging the logic of DOS with DNAbarcoding holds great promise for the identification of protein binders that can function by novel mechanisms of action. ${ }^{[8]}$

The conventional solution-phase synthesis of DELs relies on reactions that tolerate water (to keep DNA in solution) and maintain barcode integrity. ${ }^{[9,10]}$ These constraints have limited the range of transformations applicable to DEL construction and led to an enrichment of $\mathrm{sp}^{2}$-rich structures and peptidomimetics in published libraries. In response, recent work has expanded the toolbox of DEL chemists by identifying DNA-compatible conditions for established off-DNA reactions. Examples for such efforts include decarboxylative radical additions to Michael acceptors, ${ }^{[11,12]}$ Ullmann-type $N$-arylations, ${ }^{[13]}$ intramolecular nitrone cycloadditions, ${ }^{[14]}$ and $\mathrm{Ni} / \mathrm{Ir}$ dual catalytic alkylations of aryl halides. ${ }^{[15]}$ The Brunschweiger group has reported on DNA-compatible micellar catalysis ${ }^{[16]}$ and introduced an approach in the solid phase towards hexathymidine-conjugated heterocycles. ${ }^{[17,18]}$ In an alternative approach, two recent reports describe DNA-immobilization on quaternary ammonium resins to enable reactions under near anhydrous conditions including decarboxylative $\mathrm{sp}^{2}-\mathrm{sp}^{3}$ cross couplings, electrochemical aminations of aryl iodides, reductive aminations of ketones as well as copper-mediated formation of heterocycles (tin amine protocol, SnAP)..$^{[19,20]}$

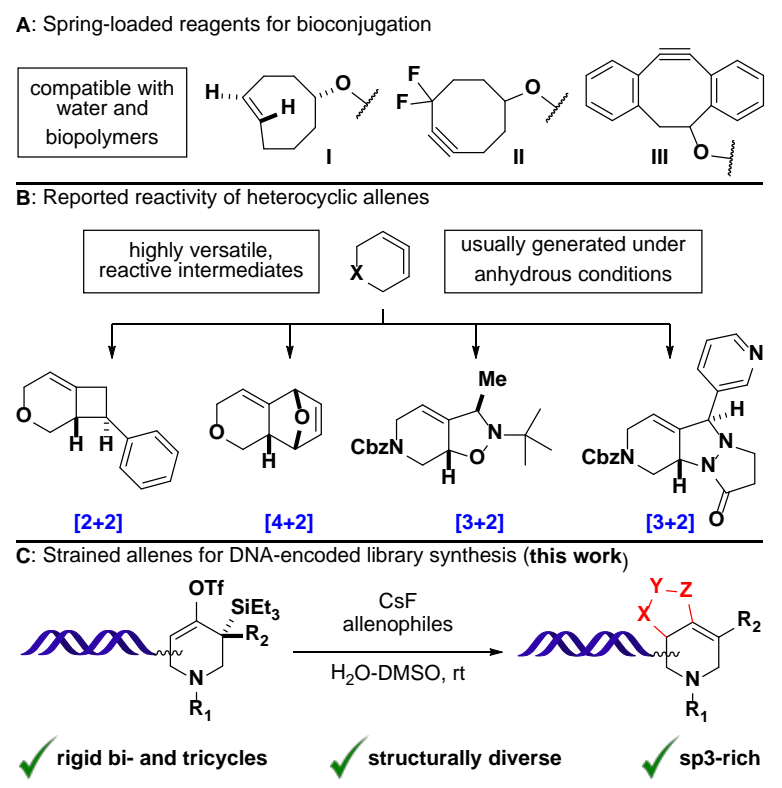

Figure 1. A: Water-compatible reagents for bioorthogonal chemistry. transCyclooctene (I) rapidly undergoes inverse-electron-demand Diels-Alder reactions with tetrazines. Cyclooctyne derivatives (II, III) undergo strain-promoted azide-alkyne cycloadditions. B: In situ generated heterocyclic allenes exhibit distinct cycloaddition modes with activated olefins, dienes and 1,3-dipoles (major diastereomers shown). C: Fluoride-induced formation of DNA-conjugated heterocyclic allenes and trapping with various allenophiles affords structurally diverse cycloaddition products.

Strain-promoted reactions have found widespread application in chemical biology. Prominent examples are the copper-free [3+2] cycloaddition of cyclooctyne-derivatives with organic azides ${ }^{[21,22]}$ and the inverse-electron-demand Diels-Alder reaction of trans-cyclooctene with tetrazines (Figure 1, A) ${ }^{[23,24]}$ Having significantly advanced the field of bioconjugation chemistry, these and related reactions are compatible with water and biopolymers by necessity. ${ }^{[25]}$ With only one recent report on inverse-electron-demand Diels-Alder reactions ${ }^{[26]}$ and reactions of cyclooctyne-DNA conjugates in the solid phase, ${ }^{[27,28]}$ strain- 
promoted reactivity has not yet found general application in the context of DELs.

When contained within 8-membered rings and smaller, cyclic allenes show increased reactivity and readily undergo strain-releasing reactions. ${ }^{[29,30]}$ Both carbo- and heterocyclic allenes have been prepared under anhydrous conditions by the action of alkyllithiums on dihalocyclopropane precursors (Doering-Moore-Skattebøl rearrangement), ${ }^{[1,32]}$ by base-induced elimination of vinylbromides, ${ }^{[33]}$ or more recently by fluoride-induced $\beta$-elimination of silyl-vinyltriflates. ${ }^{[34-36]}$ Importantly, strained allenes exhibit distinct cycloaddition modes with olefins, 1,3-dipoles, and furans $/ N$-substituted pyrroles to undergo [2+2], [3+2] and [4+2] reactions, respectively. ${ }^{[36-38]}$ The possibility to generate diverse molecular architectures from a common precursor (Figure 1, B) renders these intermediates particularly interesting in the context of diversity-oriented synthesis. Motivated by a recent report on azacyclic allene reactivity by the Garg group, ${ }^{[37]}$ we here document the successful implementation of strain-promoted cycloadditions for DNA-encoded library synthesis (Figure 1, C).

Our efforts commenced with the preparation of DNA-conjugated allene precursor 3 designed to minimally perturb steric and electronic factors of piperidine-derivative 4 used in Garg's off-DNA study. ${ }^{[3]}$ Racemic benzoic acid-derivative rac-2 was prepared in four steps from $\mathbf{1}$ closely resembling the published synthetic strategy towards 4 (see SI, Section 4c). rac-2 was elaborated into 3 by amide conjugation to a double stranded DNA-headpiece (DNA-HP) developed by researchers at GlaxoSmithKline. ${ }^{[39]}$

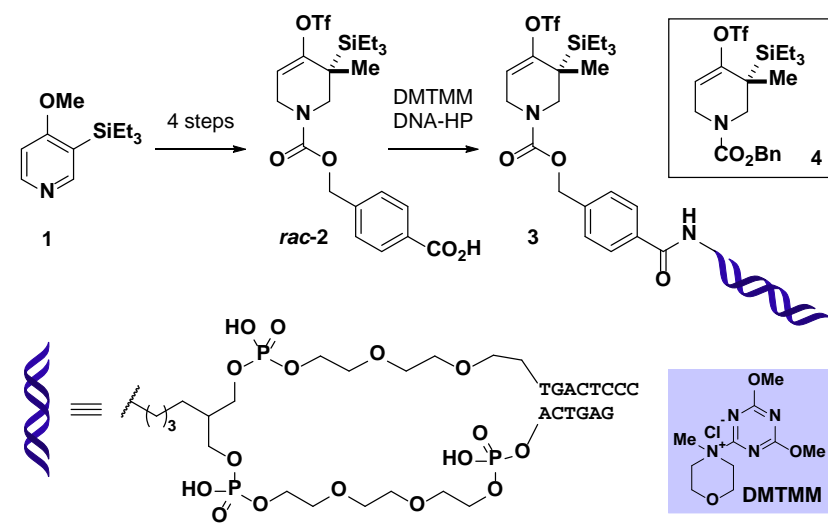

Figure 2. Preparation of on-DNA strained allene precursor $\mathbf{3}$ by conjugation of $\mathbf{r a c}-\mathbf{2}$ to DNA-headpiece (DNA-HP). For experimental details, see SI, Section 4c.

With this material in hand, on-DNA strained allene generation was investigated. To this end, 3 was combined with varying amounts of cesium fluoride and azomethine imine $\mathbf{5}$ in DMSO-water mixtures (10 $\mu \mathrm{L}$ total volume) for defined time intervals (Table 1). Following ethanol precipitation, reaction outcomes were analyzed by UPLC-MS and quantified by integration of UV absorption (260 nm), neglecting non-DNA species as judged by the absence of signal in the total ion chromatogram. In these experiments, water content emerged as a critical parameter inversely correlating with the consumption of 3 (entries 1-3). After $24 \mathrm{~h}$, significant conversion was observed only for the reaction with the lowest water content (75\% DMSO), resulting in efficient formation of a new species, the deconvoluted mass of which agreed with cycloaddition product $\mathbf{6}$. The slow conversion in presence of water, presumably originating from fluoride ion hydration, could be overcome by increasing the concentration of activating agent (entries 4-7). With further reduced water content (90\% DMSO), full consumption of $\mathbf{3}$ was observed with as little as 125 equivalents of cesium fluoride (ca. $6 \mathrm{mM}$ final concentration) within $1 \mathrm{~h}$ (entries 8 and 9). Importantly, throughout the series the only detected DNA-species were $\mathbf{3}$ and $\mathbf{6}$, provided the concentration of trapping agent 5 was sufficiently high (entries 10-12), indicating the desired transformation to be highly selective.

Table 1. Formation and trapping of a DNA-conjugated strained allenes. ${ }^{[a]}$

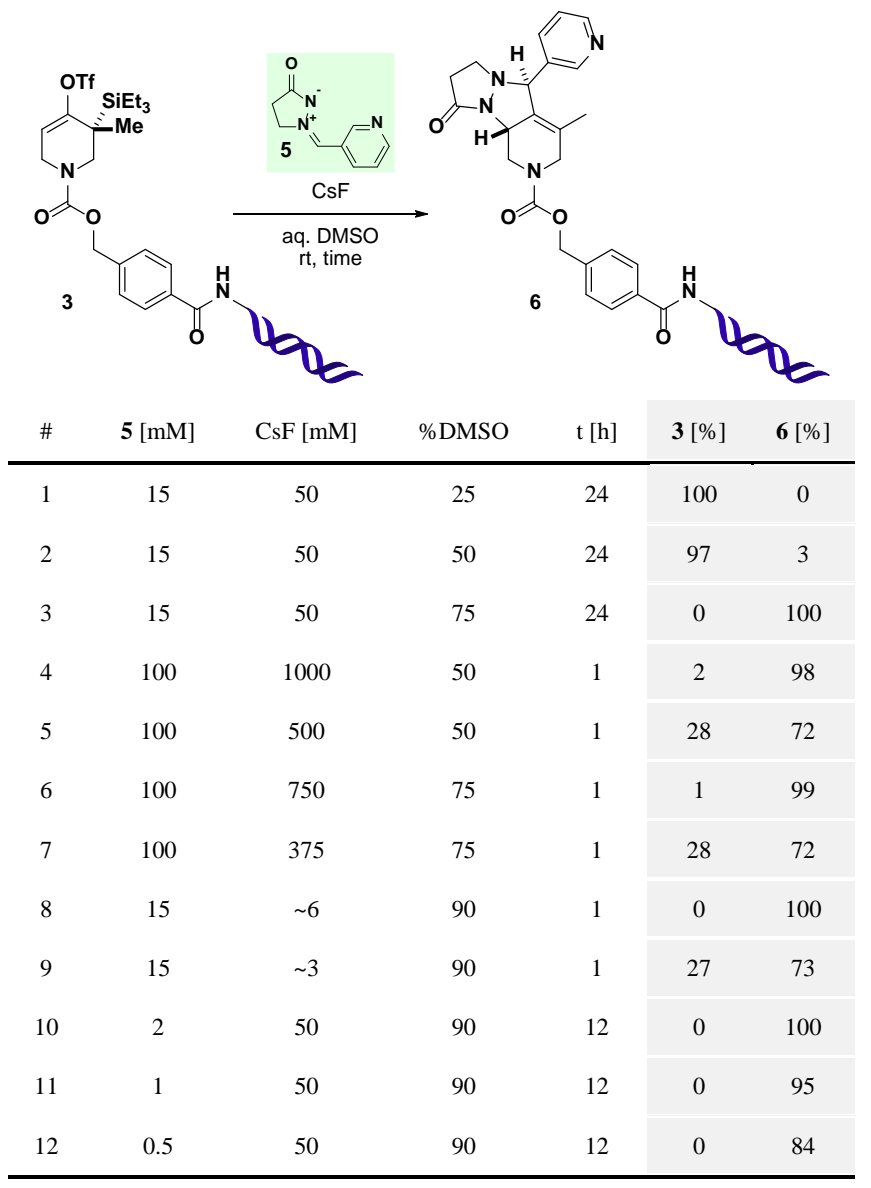

[a] Reactions were performed at room temperature with $3(0.5 \mathrm{nmol})$ in a total volume of $10 \mu \mathrm{L}$ for the indicated time. Following ethanol precipitation, residual 3 and newly formed $\mathbf{6}$ were detected by UPLC-MS and quantified (\%AUC) by integration of UV chromatograms $(260 \mathrm{~nm})$ considering DNA-species only. The relative configuration of the two newly formed stereogenic centers in $\mathbf{6}$ indicates the expected major product as observed in off-DNA precedence. ${ }^{[37]}$ For additional data, see SI, Table S1.

These initial experimental results allow for the following conclusions: 1) DNA-conjugated strained allenes can be formed in aqueous solution and exhibit considerable lifetime in the medium; 2) cycloadditions with azomethine imine 5 take place efficiently and rapidly in aqueous mixtures of DMSO (for other solvents, see SI, Table S2); 3) water content critically influences the rate of conversion (higher water content slows the reaction); 4) higher fluoride concentrations increase the rate of conversion.

Encouraged by these results, the scope of the reaction was evaluated with a variety of 1,3-dipoles, olefins and $N$-substituted pyrroles (Figure 3). In order to attenuate potential reactivity differences, building blocks were used in excess (180 mM final concentration). Nitrones derived from both aromatic and aliphatic aldehydes (7a-7d) participated in the transformation to give the expected [3+2] cycloaddition products of type 7 in high purity ( $>95 \%$ AUC). Ketone-derived nitrone $7 \mathbf{e}$ resulted in a sluggish reaction profile, presumably due to higher steric hindrance of the dipole. Pivaldehyde-derived nitrone $\mathbf{7 f}$ did not afford the expected product. Instead, a species with an apparently missing tertbutyl group was detected. This result could be explained by fast 
cycloaddition of a competing nitrone formed from small quantities of formaldehyde present in DMSO (see SI, Figure S1). [4+2] reactions of $N$-substituted pyrroles (8a-8h) efficiently afforded bridged tricyclic compounds of type $\mathbf{8}$ and tolerated the presence of numerous functional groups (nitro, aniline, phenol, aldehyde, ester). The presence of carboxylic acid 8k slowed the consumption of starting material and resulted in formation of a species exhibiting a mass in agreement with either a ketone originating from triflate hydrolysis and desilylation or an allylic alcohol resulting from hydration of the intermediate allene (req. $\mathrm{m} / \mathrm{z} 5208 \mathrm{Da}$, found $5209 \mathrm{Da}$ ). A species with the same retention time and mass spectral properties was formed in presence of excess primary amine 81. The slowed consumption of starting material in the presence of acidic protons as in $\mathbf{8 k}$ appears to be a general phenomenon (see SI, Table S3). The supposed fluoride sequestration might be explained by hydrogen bond formation and could be overcome by lowering the concentration of the acidic building block, thereby increasing the ratio of fluoride to acid (see SI, Table S4). Alternatively, fluoride sequestration may also be overcome by the addition of basic buffer (see SI, Figure S2).

[2+2] cycloadditions of commercial activated and non-activated olefins were investigated next. Vinyl (hetero-)aromatics and acrylamides including 1,1-disubstituted congeners performed very reliably in the reaction (9a-9p). Interestingly, $N$-vinyl amide $\mathbf{9 g}$ efficiently afforded the expected species while the analogous process with enol ether derivative 9v resulted in no significant product formation. This finding is in agreement with relative estimated radical stabilization energies (RSE) of initial diradical species, which are the presumed intermediates in this type of [2+2] cycloaddition. ${ }^{[30,40]}$ The difference in RSE of relevant $O$ and $\mathrm{N}$-stabilized radicals was calculated to favor the latter by ca. 25 $\mathrm{kJ} / \mathrm{mol}$ (see SI, Figure S3). ${ }^{[41]}$

The reaction with vinyl sulfone 9r afforded several species that could be only partially resolved chromatographically. Inspection of mass spectra indicated formation of multiple adducts, most likely arising from the desired [2+2] cycloaddition and additional DNA alkylation reactions (up to seven events by mass spectrometry (see SI, Figure S4). This finding prompted us to examine a variety of acceptor-substituted olefins of varied electrophilicity on the Mayr reactivity scale ${ }^{[42]}$ (see SI, Table S5). Qualitative correlation of the reaction outcomes with the corresponding electrophilicity parameters of building blocks revealed that electrophiles with $E_{\mathrm{DMSO}}>-19$ tend to undergo DNA alkylation reactions under the chosen conditions (90\% aq. DMSO, $180 \mathrm{mM}$ building block, $200 \mathrm{mM}$ CsF, $1 \mathrm{~h}, \mathrm{rt}$ ). Less electrophilic building blocks did not show signs of DNA alkylation. Although observed in 90\% aq. DMSO, these findings might have implications not only in the context of DNA-encoded library synthesis, but for the field of bioconjugate chemistry in general, where maleimides $\left(E_{\text {DMso }} \approx-14\right)$ are often used, as well as for toxicological assessment of electrophilic drugs. 

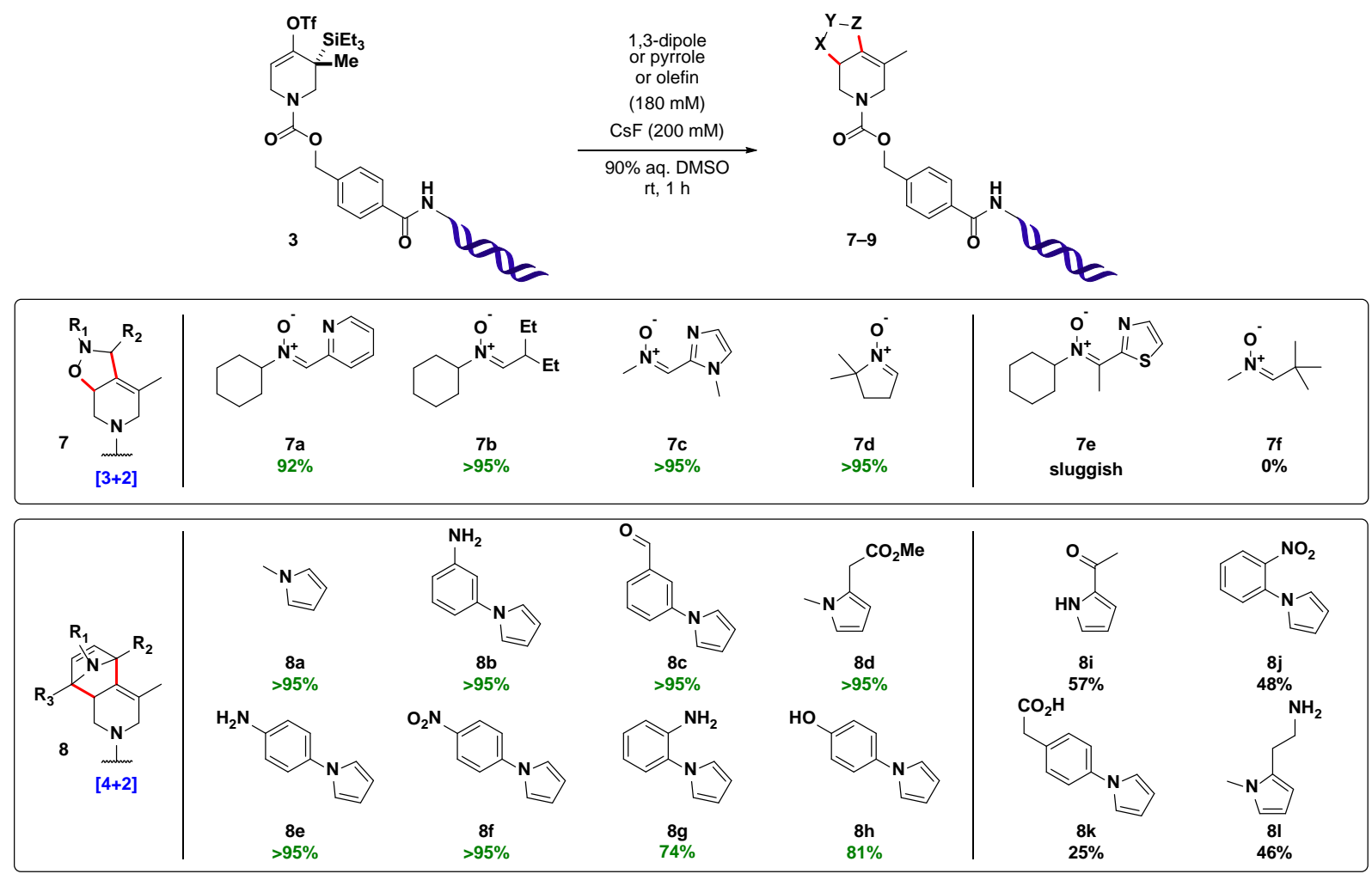

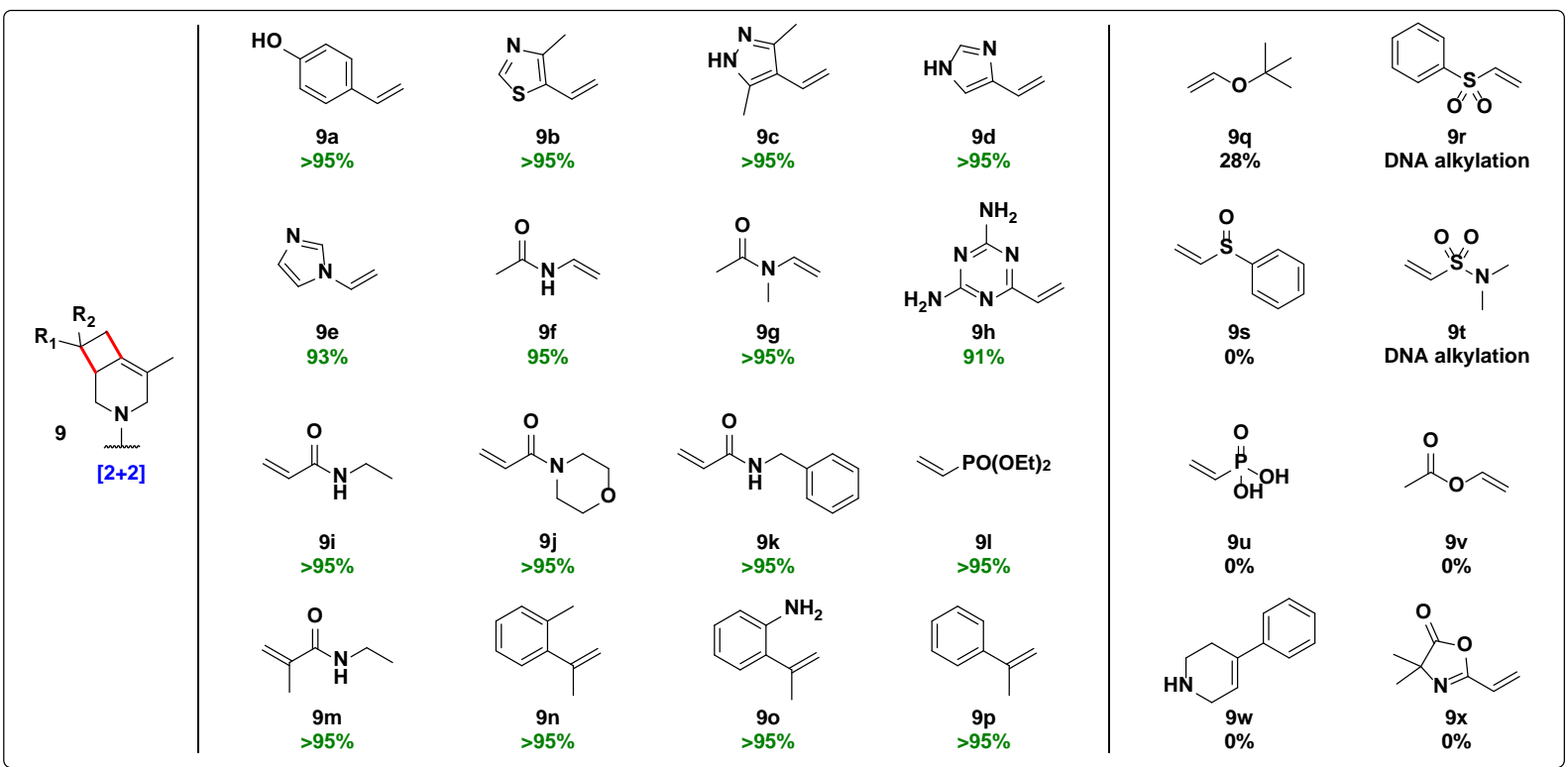

Figure 3. Selected examples of building blocks used in strain-promoted cycloaddition reactions of DNA-conjugated allenes derived in situ from 3. Product display (\%AUC) was determined by integration of UV signals ( $260 \mathrm{~nm}$ ) considering DNA-species only. For additional examples of [2+2] reactions, see SI, Figure S5. For titration experiments with each type of cycloaddition partner, see SI, Figure S6.

Unlike reagent classes such as boronic acids, aldehydes or amines, the number of commercially available 1,3-dipoles is limited. Thus, a combinatorial synthesis of such building blocks ideally avoiding tedious purification would be highly desirable. We therefore attempted the synthesis of a test set of azomethine imines by simply combining 3pyrazolidinone with various aldehydes in ethanol. Incubation of the resulting mixtures at room temperature overnight, removal of the volatiles and reconstitution of residual material in DMSO afforded solutions for immediate use in on-DNA reactions, notably without purification. Assuming quantitative azomethine imine formation, onDNA precursor 3 was combined with 1,3-dipoles (15 mM final concentration) and cesium fluoride (50 mM final concentration) in $90 \%$ aqueous DMSO. In this non-optimized procedure, about half of the building block solutions thus prepared validated ( $>80 \%$ AUC) to afford the expected DNA-species as identified by UPLC-MS analysis following ethanol precipitation (see SI, Figure S7). 


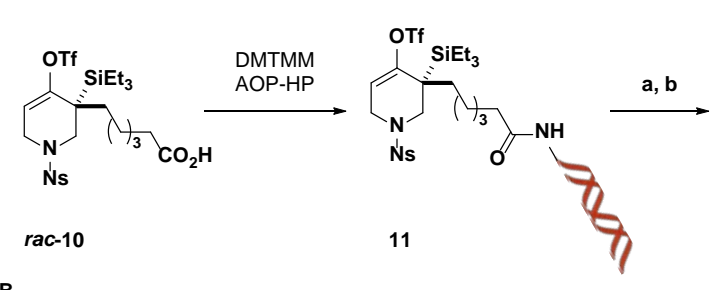

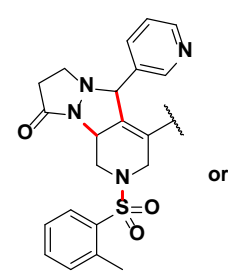

12

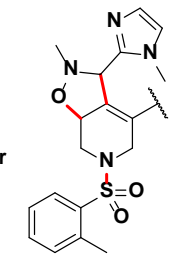

13

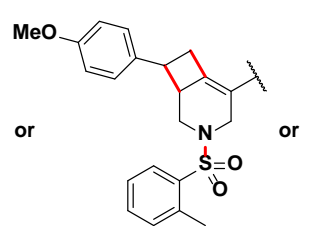

14

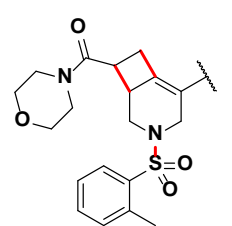

15

B

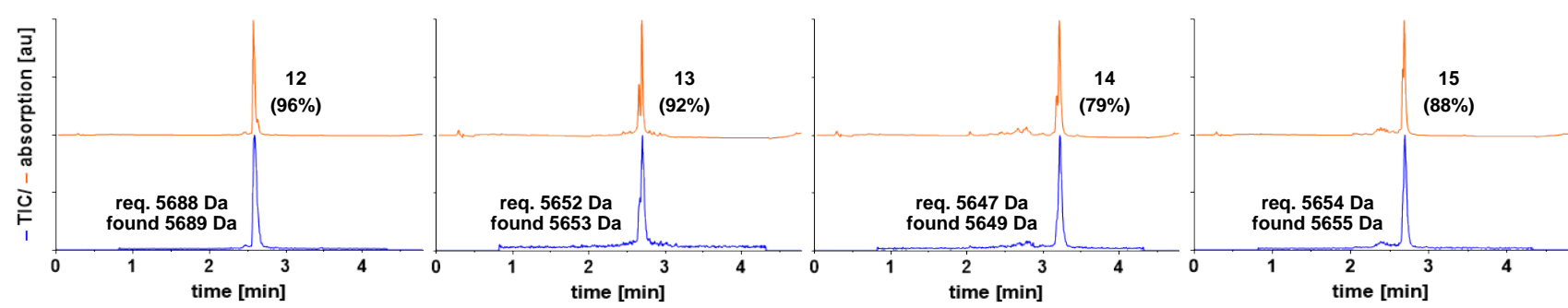

Figure 4. A: Synthesis of DNA-conjugated strained allene precursor 11 enables two-step diversification. Reagents and conditions: a) 11 (5 nmol), 1,3-dipole or styrene derivative (25 mM), CsF (23 mM), 90\% aq. DMSO, rt, $1 \mathrm{~h}$; then 4-methoxythiophenol (85 mM), carbonate buffer (pH10), 60\% aq. DMSO, $80^{\circ} \mathrm{C}, 1 \mathrm{~h}$. For UPLC-MS analysis of intermediate piperidines, see SI, Figure S8; b) 2-Methylbenzenesulfonyl chloride (40 mM), phosphate buffer (pH8), 20\% aq. MeCN, rt, $12 \mathrm{~h}$. B: UPLC-MS analysis of $N$-sulfonylation reactions following EtOH precipitation indicates efficient formation of compounds 12-15 (orange: UV ( $260 \mathrm{~nm}$ ), blue: total ion chromatogram). Figures in parentheses refer to \%AUC of the corresponding species.

While DNA-conjugate $\mathbf{3}$ was shown to undergo fluoride-induced elimination readily to form a highly reactive strained allene that could be trapped even in aqueous media, it is amenable to one-step diversification only-provided no additional functionalities are introduced by virtue of the cycloaddition partners. To highlight the promise of the described transformation in terms of combinatorial DEL synthesis, a second-generation substrate (11) was prepared by conjugating rac-10 to a PEG-linker extended version of DNA-HP (AOP-HP) via amide bond formation. 11 exhibits a nosyl group on nitrogen, the on-DNA deprotection of which has been described, ${ }^{[43]}$ and as such offers two points of diversification (strained allene cycloaddition, $N$-capping). In addition, library synthesis should benefit from spatial separation of the nucleophilic nitrogen and the cycloaddition product appendages, providing more uniform reactivity during $N$-capping reactions. Figure 4 shows four examples of a synthetic sequence involving strain-promoted cycloadditions/Ns-deprotection to afford intermediate piperidines, which were subjected to $N$-sulfonylations using 2-methylbenzenesulfonyl chloride as a representative $N$-capping reaction (Figure 4, A). Final products 12-15 were derived in 79$96 \%$ AUC as evident from the UV chromatograms (Figure 4, B).

In accord with off-DNA precedence, ${ }^{[38,44,45]}$ we note that the heredescribed on-DNA transformations are expected to form diastereoisomeric mixtures of varying ratios. Indeed, the corresponding UV chromatograms regularly show split peaks (see Figure 4, B and SI) sharing the same mass spectral properties. We acknowledge that this property of the presented on-DNA process may complicate off-DNA hit validation following a library screening campaign but advocate for its widespread use given the unprecedented nature of the formed products. In this context, a substructure search of cores 7-9 in the ChEMBL database returned zero hits, ${ }^{[46]}$ supporting the notion that derivatives of these structures are indeed covering uncharted chemical space.

In an additional experiment, aliquots of the intermediate piperidineDNA conjugates, isolated and purified by standard ethanol precipitation, were subjected to T4 DNA ligase-mediated ligation reactions with a 23basepair primer sequence. The success of these ligations was confirmed by gel electrophoretic analysis (see SI, Figure S9). Furthermore, realtime polymerase chain reaction (qPCR) studies were performed to assess the extent of potential DNA degradation during the reaction. ${ }^{[9]}$ To this end, a full-length DNA-encoded library was subjected to conditions with varied DMSO and cesium fluoride content (see SI, Figure S10). Subsequent quantification of amplifiable material did not indicate any DNA degradation in samples treated with CsF (100 mM) in 85\% aq. DMSO. Samples incubated with very high fluoride concentrations (4 M $\mathrm{CsF}$ in $50 \%$ aq. DMSO or $3 \mathrm{M} \mathrm{CsF}$ in $75 \%$ aq. DMSO) showed ca. $80 \%$ remaining amplifiable material. Notably, all test conditions in this degradation study used substantially more activating agent than needed to enable efficient allene formation (see Table 1 and SI, Table S1). In conclusion, the successful outcome of enzymatic ligation reactions with samples that underwent strain-promoted cycloadditions along with negligible DNA degradation warrants a promising integration of the described transformation into existing DEL synthesis workflows.

The present work describes a rare example of a DNA-compatible process allowing the synthesis of highly structurally diverse, rigid core structures of high $\mathrm{sp}^{3}$-content from a single precursor. Extension of the concept to other strained allene precursors including bicyclic and sevenmembered systems may further expand the scope of unprecedented structures that can be incorporated into DNA-encoded libraries. Given the convenient reaction setup, the remarkably efficient and selective reaction profiles and the number of commercially available or easily prepared building blocks, strain-promoted cycloaddition reactions should find widespread use in the field of DNA-encoded library synthesis.

\section{Acknowledgements}

John Capece, Jennifer Poirier, Philip Michaels, Carmelina Rakiec, Thomas Dice and Ritesh Tichkule are gratefully acknowledged for excellent technical and analytical support. Bruce Hua and Drs. Christopher Gerry, Wenyu Wang, Christian Gampe, Simone Bonazzi, Nichola Smith and Frédéric Berst are gratefully acknowledged for general support, fruitful discussions and valuable feedback during preparation of this manuscript.

\section{Notes}

The authors declare the following competing financial interest(s): S.L.S serves on the Board of Directors of the Genomics Institute of the Novartis Research Foundation (“GNF”); is a shareholder and serves on the Board of Directors of Jnana Therapeutics; is a shareholder of Forma 
Therapeutics; is a shareholder and advises Decibel Therapeutics and Eikonizo Therapeutics; serves on the Scientific Advisory Boards of Eisai Co., Ltd., Ono Pharma Foundation, Exo Therapeutics, and F-Prime Capital Partners; and is a Novartis Faculty Scholar.

Keywords: DNA-encoded library • diversity-oriented synthesis • strained allene $\bullet$ cycloaddition $\bullet$ combinatorial chemistry

[1] D. Neri, R. A. Lerner, Annu. Rev. Biochem. 2018, 87, 479

[2] A. L. Satz, ACS Med. Chem. Lett. 2018, 9, 408

[3] W. G. Cochrane, M. L. Malone, V. Q. Dang, V. Cavett, A. L. Satz, B. M. Paegel, ACS Comb. Sci. 2019, 21, 425.

[4] A. B. MacConnell, A. K. Price, B. M. Paegel, ACS Comb. Sci. 2017, 19, 181

[5] W. R. J. D. Galloway, A. Isidro-Llobet, D. R. Spring, Nat. Commun. 2010, 1, 80.

[6] P. A. Clemons, J. A. Wilson, V. Dančík, S. Muller, H. A. Carrinski, B. K. Wagner, A. N. Koehler, S. L. Schreiber, PNAS 2011, 108, 6817.

[7] C. J. Gerry, S. L. Schreiber, Nat. Rev. Drug Discov. 2018, 17, 333.

[8] S. L. Schreiber, Isr. J. Chem. 2019, 59, 52.

[9] A. S. Ratnayake, M. E. Flanagan, T. L. Foley, J. D. Smith, J. G. Johnson, J. Bellenger, J. I. Montgomery, B. M. Paegel, ACS Comb. Sci. 2019, 21, 650

[10] M. L. Malone, B. M. Paegel, ACS Comb. Sci. 2016, 18, 182

[11] J. Wang, H. Lundberg, S. Asai, P. Martín-Acosta, J. S. Chen, S. Brown, W. Farrell, R. G. Dushin, C. J. O’Donnell, A. S. Ratnayake, et al., PNAS 2018 115, E6404.

[12] D. K. Kölmel, R. P. Loach, T. Knauber, M. E. Flanagan, ChemMedChem 2018, 13, 2159.

[13] Y. Ruff, F. Berst, Med. Chem. Commun. 2018, 9, 1188.

[14] C. J. Gerry, Z. Yang, M. Stasi, S. L. Schreiber, Org. Lett. 2019, 21, 1325.

[15] J. P. Phelan, S. B. Lang, J. Sim, S. Berritt, A. J. Peat, K. Billings, L. Fan, G. A. Molander, J. Am. Chem. Soc. 2019, 141, 3723

[16] M. K. Škopić, K. Götte, C. Gramse, M. Dieter, S. Pospich, S. Raunser, R. Weberskirch, A. Brunschweiger, J. Am. Chem. Soc. 2019, 141, 10546.

[17] M. Potowski, V. B. K. Kunig, F. Losch, A. Brunschweiger, Med. Chem. Commun. 2019, 10, 1082

[18] M. K. Škopić, H. Salamon, O. Bugain, K. Jung, A. Gohla, L. J. Doetsch, D. dos Santos, A. Bhat, B. Wagner, A. Brunschweiger, Chem. Sci. 2017, 8, 3356

[19] Y. Ruff, R. Martinez, X. Pellé, P. Nimsgern, P. Fille, M. Ratnikov, F. Berst, 2019, DOI 10.26434/chemrxiv.8174864.v4.
[20] D. T. Flood, S. Asai, X. Zhang, J. Wang, L. Yoon, Z. C. Adams, B. C. Dillingham, B. B. Sanchez, J. C. Vantourout, M. E. Flanagan, et al., J. Am Chem. Soc. 2019, 141, 9998.

[21] N. J. Agard, J. A. Prescher, C. R. Bertozzi, J. Am. Chem. Soc. 2004, 126, 15046 .

[22] J. M. Baskin, J. A. Prescher, S. T. Laughlin, N. J. Agard, P. V. Chang, I. A. Miller, A. Lo, J. A. Codelli, C. R. Bertozzi, PNAS 2007, 104, 16793.

[23] M. L. Blackman, M. Royzen, J. M. Fox, J. Am. Chem. Soc. 2008, 130, 13518

[24] K. Lang, L. Davis, S. Wallace, M. Mahesh, D. J. Cox, M. L. Blackman, J. M. Fox, J. W. Chin, J. Am. Chem. Soc. 2012, 134, 10317.

[25] J. A. Prescher, C. R. Bertozzi, Nat. Chem. Biol. 2005, 1, 13.

[26] H. Li, Z. Sun, W. Wu, X. Wang, M. Zhang, X. Lu, W. Zhong, D. Dai, Org. Lett. 2018, 20, 7186.

[27] I. Singh, C. Freeman, F. Heaney, Eur. J. Org. Chem. 2011, 6739.

[28] I. Singh, F. Heaney, Chem. Commun. 2011, 47, 2706.

[29] K. J. Daoust, S. M. Hernandez, K. M. Konrad, I. D. Mackie, J. Winstanley, R. P. Johnson, J. Org. Chem. 2006, 71, 5708.

[30] M. Christl, in Modern Allene Chemistry, John Wiley \& Sons, Ltd, 2008, 243.

[31] W. R. Moore, W. R. Moser, J. Am. Chem. Soc. 1970, 92, 5469.

[32] W. R. Moore, W. R. Moser, J. Org. Chem. 1970, 35, 908.

[33] G. Wittig, P. Fritze, Angew. Chem. Int. Ed. 1966, 5, 846.

[34] V. A. Lofstrand, F. G. West, Chem. Eur. J. 2016, 22, 10763.

[35] I. Quintana, D. Peña, D. Pérez, E. Guitián, Eur. J. Org. Chem. 2009, 2009 5519.

[36] J. S. Barber, E. D. Styduhar, H. V. Pham, T. C. McMahon, K. N. Houk, N. K. Garg, J. Am. Chem. Soc. 2016, 138, 2512.

[37] J. S. Barber, M. M. Yamano, M. Ramirez, E. R. Darzi, R. R. Knapp, F. Liu, K. N. Houk, N. K. Garg, Nat. Chem. 2018, 10, 953.

[38] M. Schreck, M. Christl, Angew. Chem 1987, 99, 720.

[39] M. A. Clark, R. A. Acharya, C. C. Arico-Muendel, S. L. Belyanskaya, D. R. Benjamin, N. R. Carlson, P. A. Centrella, C. H. Chiu, S. P. Creaser, J. W. Cuozzo, et al., Nat. Chem. Biol. 2009, 5, 647.

[40] E. A. Boafo, K. Darko, B. A. Afriyie, R. Tia, E. Adei, J. Mol. Graph. Model. 2018, 81, 1 .

[41] J. Hioe, H. Zipse, Org. Biomol. Chem. 2010, 8, 3609.

[42] D. S. Allgäuer, H. Jangra, H. Asahara, Z. Li, Q. Chen, H. Zipse, A. R. Ofial, H. Mayr, J. Am. Chem. Soc. 2017, 139, 13318.

[43] T. Franch, M. D. Lundorf, S. Jakobsen, E. K. Olsen, A. L. Andersen, A. Holtmann, A. H. Hansen, A. M. Sørensen, A. Goldbech, D. De Leon, et al. (Nuevolution A/S), WO/2007/062664, 2007.

[44] M. Christl, M. Braun, Chem. Ber. 1989, 122, 1939.

[45] M. Yamano, R. Knapp, A. Ngamnithiporn, M. Ramirez, K. Houk, B. Stoltz, N. K. Garg, Angew. Chem. Int. Ed. 2019, 58, 5653.

[46] ChEMBL database accessed on Nov. $9^{\text {th }}$ under https://www.ebi.ac.uk/chembl/ 